\title{
Coordinate Fuzzy Transforms and Fuzzy Tent Maps - Properties and Applications
}

\author{
Horia-Nicolai L. TEODORESCU ${ }^{1,2}$ \\ ${ }^{1}$ Romanian Academy - Iasi Branch, Iasi, Romania \\ 2 'Gheorghe Asachi' Technical University of Iasi, \\ 67, D. Mangeron, Iasi, Romania
}

\begin{abstract}
The paper introduces transforms of the coordinates in the Euclidean space based on fuzzy logic systems and investigates properties of these transforms. Tents maps modified using coordinate fuzzy transforms are introduced as a direct application of the fuzzy transforms. For brevity, only line and plane fuzzy transforms are considered.
\end{abstract}

Keywords: fuzzy transformation, real line, Euclidean space, tent map, nonlinear transformation, histogram equalization.

\section{Introduction}

This section recalls basic facts, sometimes seen from a different perspective. In defining fuzzy sets on the real axis, typically two steps are involved; the first connects the line or a segment of it with a finite set $\Lambda$ of linguistic descriptions (labels), $\left\{\boldsymbol{\lambda}_{j}\right\}_{j=1, \ldots, N}$ (such as in 'a tall man'); the second assigns (one-to-one) intervals of the line, $J_{j}$, to the linguistic labels, $\lambda_{j} \leftrightarrow J_{j}$, where typically each interval is overlapping with at least two other intervals. To each interval $J_{j}$ one assigns a function, named membership function (m.f.). The m.f.s are defined on the line, positive, and normalized (i.e., with maximal value equal to 1 ); in addition, they are null valued everywhere except (at most) the specified intervals. Thus, a set of functions $M=\left\{\mu_{j}\right\}_{j=1, \ldots, N}$ is put into one-to-one correspondence with the set of intervals and respectively to the linguistic labels, $\mu_{j} \leftrightarrow J_{j} \leftrightarrow \lambda_{j}$. In this way, to every point of the real line, $x \in \cup_{j} J_{j}$, one associates a subset of membership functions overlapping in $x$, by the condition $\mu(x) \neq 0$. Let us denote this subset by $M_{x}=\left\{\mu_{j} \mid \mu_{j}(x) \neq 0\right\}$. This entire construction corresponds to the fuzzification of the real line (or of an interval of it.)

A second construction, which reverses the above one, is stereotypically as follows. Assume that all m.f.s satisfy the condition that they have value 1 at exactly one point; denote that point by $a_{j}$. For every $x$, assign to the points $a_{j}$ a weight $w_{j}(x)=\mu_{j}(x)$. In this way, every value of the real line is endowed with the set of couples $\left\{\left(a_{j}, \mu_{j}(x)\right)\right\}_{j}$.
All $a_{j}$ points corresponding to m.f.s not in $M_{x}$ have null weights. Next, assign to $x$ the weighted average of the points $a_{j}$, $x^{\prime}(x)=\sum_{j} a_{j} \mu_{j}(x) / \sum_{j} \mu_{j}(x)$. This is equivalent with defining a Takagi-SugenoKang (TSK) system from $\mathbf{R}$ to itself; the last operation corresponds to the defuzzification.

The entire construction, composed of the above two constructions, when applied to $R$, i.e., when $\mathbf{R}$ is fuzzified and then mapped on itself with a TSK, will be named type I coordinate fuzzy transformation (CoFT) of the real line. The construction is easily extended to $n$ dimensional Euclidean spaces. The transformation corresponds to the TSK fuzzy systems, where the output of the system is the same space as the input. The second type of fuzzy transformation of the space follows the idea of Mamdani, assigning a second set of functions $\eta_{h}$ to the real line and next assigning to every $\mu_{k}$ at least one $\eta_{h}$ (i.e., providing the 'rules' of the FLS). The 'reverse' construction is finalized according to defuzzification for Mamdani systems.

The above discussion was meant to show why the name of fuzzy transformation of the real line is used for these constructions. The investigation of the issue of fuzzy transforms is useful, among others, because any inverse model based controller can be seen as a space transform of the input space of the controller by the reverse model. The paper studies several examples of fuzzy space transforms and potential applications.

The second Section introduces the definitions and properties of the fuzzy transforms, while 
the third Sections presents fuzzy tent maps defined by means of the transforms. Section 4 shows an example of CoFT in prediction. The last Section concludes the paper.

The following abbreviations are used throughout the paper: m.f.(s) - membership function(s), FL - fuzzy logic, FLS - fuzzy logic system, TSK - Takagi-Sugeno-Kang FLS, CoFT - coordinate fuzzy transform.

\section{Definitions, Properties and Examples of Fuzzy Transforms}

Definition. We name space fuzzy transform of the interval $J$ in the $n$-th dimensional Euclidean space an $n$-dimensional subspace $H=\psi(J)$, $H \in R^{n}$, where the transform $\psi$ denotes an $n$ input, $n$-output fuzzy system with defuzzification on each output.

In the remaining part of the paper, only TSK fuzzy systems will be used for $\psi$. A very simple case of fuzzy transform, although an important one is the identity transform given in Property 1. Translations and scaling (linear deformation) along an axis are easily built based on the discussion in the proof of Property 1. Example of nonlinear transforms of the axis ("nonlinear first bisectors') will be discussed in detail.

Property 1. A TSK system with input variable $x$ and output variable $x^{\prime}$ and having the rules If $x$ is $\tilde{A}_{k}$ then $\tilde{x}^{\prime}=\tilde{x}_{k} \quad(k=1, \ldots, \mathrm{n})$, where $\tilde{A}_{k}$ is a fuzzy set with isosceles triangular m.f. with center in $x_{k}$ and $\tilde{x}_{k}$ is the singleton in $x_{k}$ performs the identical transform $x^{\prime}=x$ where $x^{\prime}$ is the defuzzified output.

Proof. A TSK system with isosceles triangular membership functions at the input is linear piecewise. Because $\tilde{x}_{k}$ is the singleton in $x_{k}$, the TSK output is interpolator through the points $\left(x_{k}, x_{k}\right)$. Because it is a linear interpolator, it results that it is the identical transform on $\left[x_{1}, x_{n}\right]$.

Fuzzify the first diagonal, $x=y$ as follows. The $O x$ axis is fuzzified by defining single mode m.f., $\mu_{k}(x), \mu_{k}\left(a_{k}\right)=1$. Assume that at most three m.f.s overlap in any $x$. Then, for a specified $x$, the $O x$ axis transformation (deformation) is $x^{\prime}=h(x)$ defined by, for example, as

$$
\begin{aligned}
& x \rightarrow x^{\prime}=h(x)= \\
& =\frac{a_{k-1} \mu_{k-1}(x)+a_{k} \mu_{k}(x)+a_{k+1} \mu_{k+1}(x)}{\mu_{k-1}(x)+\mu_{k}(x)+\mu_{k+1}(x)}
\end{aligned}
$$

With this 'new' $x$, the function $y=f(x)$ can be computed as usually. Thus, if we have a classic controller and wish to adjust it locally, we can apply simply a transformation of the input axis and then apply it to the controller. Again, this transformation can be seen as a 'system inverse model', because the control, including the transformation effect, is $f(h(x))$.

When the m.f.s are all triangular (possibly not isosceles), $h(x)=x$, so this case is the identity transformation, of little interest. The same happens when all m.f.s are complementary on their intervals of overlapping, in the sense that $\mu_{k-1}(x)=1-\mu_{k}(x)$ and $\mu_{k}(x)=1-\mu_{k+1}(x)$ whenever $\mu_{k}(x)>0$. Notice that complementarity does not imply that the membership functions are triangular or piecewise linear.

Assume a uniform sampling of the $x$ space, i.e., $a_{k}-a_{k-1}=c$. Also, assume that the m.f.s are not complementary and that they are parabolic, that is, piece-wisely they are branches of parabolas,

$$
\begin{aligned}
& \mu_{k}(x)= \\
& \begin{cases}\left(x-a_{k-1}\right)^{2} / c^{2} & \text { for } a_{k-1} \leq-x \leq a_{k} \\
\left(x-a_{k+1}\right)^{2} / c^{2} & \text { for } a_{k} \leq-x \leq a_{k+1}\end{cases}
\end{aligned}
$$

When the $x$-space is not uniformly sampled and the membership functions are chosen parabolic at left and right of the vertex, i.e. (see Figure 1),

$$
\mu_{k}(x)=\left\{\begin{array}{l}
\frac{\left(x-a_{k-1}\right)^{2}}{\left(a_{k}-a_{k-1}\right)^{2}}, a_{k-1} \leq x \leq a_{k} \\
\frac{\left(x-a_{k+1}\right)^{2}}{\left(a_{k+1}-a_{k}\right)^{2}}, a_{k} \leq x \leq a_{k+1}
\end{array}\right.
$$

Because the left and right sides of the membership functions are parabola arcs, the m.f.s will be named parabolic, respectively semi-parabolic in case the space sampling is not uniform.

In case of uniform sampling of the $x$ space, assuming, as usually, that successive m.f.s overlap on $\left[a_{k}, a_{k+1}\right]$ and that $\mu_{k}\left(a_{k}\right)=1$, and taking into account that $a_{k+1}-a_{k}=a_{k}-a_{k-1}$, one obtains on $\left[a_{k-1}, a_{k+1}\right]$ the transform

$$
h(x)=\frac{a_{k} \mu_{k}(x)+a_{k+1} \mu_{k+1}(x)}{\mu_{k}(x)+\mu_{k+1}(x)}
$$




$$
\begin{aligned}
& \theta(x)= \\
& \left\{\begin{array}{l}
\alpha+s_{1}\left(x-v_{0}\right), s_{1}>0, \text { for } v_{0} \leq x \leq v_{1} \\
\beta+s_{2}\left(x-v_{1}\right), s_{2}<0, \text { for } v_{1} \leq x \leq v_{2}
\end{array}\right.
\end{aligned}
$$

Figure 1 illustrates a set of parabolic m.f.s and the 'fuzzy identity' transform obtained with the respective parabolic m.f.s, according to the above formulas. Replacing in (5) $a_{k}=a_{0}+k c$ and $a_{k+1}=a_{0}+(k+1) c$, one obtains a somewhat closer form.

One easily modifies the above transform using different m.f.s, for example quadratic, such as

$$
\mu_{k}(x)=\left\{\begin{array}{l}
\frac{\left(x-a_{k-1}\right)^{4}}{\left(a_{k}-a_{k-1}\right)^{4}}, a_{k-1} \leq-x \leq a_{k} \\
\frac{\left(x-a_{k+1}\right)^{4}}{\left(a_{k+1}-a_{k}\right)^{4}}, a_{k} \leq-x \leq a k+1
\end{array}\right.
$$

The graph of the membership functions (6) is shown in Figure 1 (a).

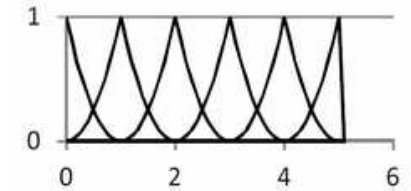

(a)

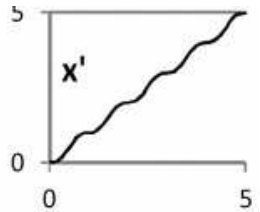

(b)
Figure 1. (a) Uniformly spaced interval of the variable and the corresponding parabolic membership functions. (b) Pseudo-identity transformation - transformation of the first bisector.

When the axis $O x$ is replaced with the axis $O x^{\prime}$, where $x^{\prime}=h(x)$, the above represents the fuzzy transform of the coordinate axis.

Properties. The deformation has the following properties:

1. The transforms are nonlinear, namely piecewise rational transformations when the membership functions are polynomial.

2. CoFT (5) and similar ones preserve the points $a_{k}$, that is, it is an interpolation of the $O x$ axis in these points;

3. The minimal deformation occurs at the points $a_{k}$, (where the derivative is minimal), while the maximal deformations, for uniformly sampled spaces, occur at $\frac{a_{k}+a_{k+1}}{2}$

4. When the sampling of the $O x$ axis is uniform (i.e., when the distance between $a_{k}$ and $a_{k+1}$ is constant for all $k$ ), the transform is periodical.
Proof. (1) and (2) result directly from the definition equations. The proof of (3) and (4) also results from the definition equations, after elementary calculus.

In many applications, a logarithmic scale is preferred, where $a_{k}=10^{k}$. Then, the semiparabola in the above definition of the m.f.s should be changed accordingly. Observe that nothing prevents the choice of 'inverse' semiparabolas, $\quad 1-\left(x-a_{k}\right)^{2} / c^{2}$ or of other functions suitable for m.f.s and for the desired deformation of the real line.

Because the above transform iterates the first diagonal of the space $\left(x, x^{\prime}\right)$ through the values $a_{k}$, it will be named fuzzy pseudoidentity transformation.

It is worth to mention that by applying first CoFTs and then standard FLSs, which thus 'see' the transformed input space, more powerful equivalent FLSs are built. The values $a_{k}$ and the type (parabolic, quadratic etc.) of the membership functions in the CoFT define the transform and can be modified (adjusted) in adaptive CoFT-FLSs to suit specific applications.

The transformation defined in this Section uses a different approach and is completely distinct from the one with resembling name introduced and studied in [3], [10-14], although the application fields of these transforms may overlap.

\section{CoFT-based Fuzzy Tent Maps}

Chaotic processes are ubiquitous in nature, including physics, chemistry and biology. Moreover, they occur in every major system related to humans, including economic and social systems, e.g. [7, 8]. As a consequence, a vast literature deals with the modeling of the nonlinear dynamic phenomena using crisp [2, $15,16,17]$ and fuzzy $[18,19]$ approaches, and with computer methods for simulating them, e.g. $[1,21]$.

Tent maps [2, 8] are defined as piecewise linear functions with only two branches, the first increasing and the second decreasing. It will be assumed subsequently that both branches have values in the same interval $[\alpha, \beta]$, that is

$$
\begin{aligned}
& \theta(x)= \\
& \left\{\begin{array}{l}
\alpha+s_{1}\left(x-v_{0}\right), s_{1}>0, \text { for } v_{0} \leq x \leq v_{1} \\
\beta+s_{2}\left(x-v_{1}\right), s_{2}<0, \text { for } v_{1} \leq x \leq v_{2}
\end{array}\right.
\end{aligned}
$$


where $\quad \alpha+s_{1}\left(v_{1}-v_{0}\right)=\beta \quad$ and $s_{2}=-(\beta-\alpha) /\left(v_{2}-v_{1}\right)$; most frequently, $s_{1}=-s_{2}=2$ [2]. In (7), $\alpha+s_{1}\left(x-v_{0}\right)$ is the increasing linear section of the tent map and $\beta+s_{2}\left(x-v_{1}\right)$ is the decreasing one.

Tent maps, which are kin to logistic and cubic maps, are simple yet useful examples of functions that, by iteration, produce chaotic time series when the function graph intersects the first bisector of the plane $[2,8]$. The class of tent maps (7), on a specified interval, $\left[v_{0}, v_{2}\right]$, includes functions with a single parameter, namely the position of the tent peak, $v_{1}$. This limits their suitability for modelling processes that have a large number of parameters, as well as their flexibility in fitting the model.

On the other hand, the simplicity of the tent maps and their scarcity of adjustable parameters, which hampers their use in modelling and prediction applications, make them computationally effective in chaotic series generation. As a consequence, tent maps have been studied in applications for secure communications and data encryption [23, 24] and may be used to replace other chaotic systems in applications such as the ones described in [23, 24]. A combination of CoFT with a crisp tent map could be a compromise between computational affordability and modelling power. Next, the use of the transform in combination with the tent map is studied.

The first combination investigated is

$$
y_{n}=\theta\left(h\left(x_{n-1}\right)\right), x_{n}=y_{n}, n \geq 1
$$

with the transform included in the iteration, and with a specified initial condition $x_{0}$. The system described by (8) will be named (iterated) fuzzy tent map because the CoFT $h$ is part of the iteration, such as the tent map 'sees' a fuzzy transformed space of the crisp input.

Next, we are interested in the iteration

$$
y_{n}=\theta\left(x_{n-1}\right), x_{n}=y_{n-1}, y_{n}^{*}=g\left(y_{n}\right) \text {, }
$$

where the iteration produces $y_{n}^{*}$. In the last case, the transform is applied to the 'output' of the tent map and is not included in the iteration. This case is less interesting than the one described by (8).

Using (8) and combining the equation (7) of the crisp tent map with the fuzzy space transformation, one obtains,

$$
\begin{aligned}
& x_{n+1}=h\left(y_{n}\right)=\sum_{j} a_{j} \mu_{j}\left(y_{n}\right) / \sum_{j} \mu_{j}\left(y_{n}\right), \\
& y_{n+1}=\theta\left(x_{n+1}\right)
\end{aligned}
$$

For example, considering the transformation with equally sampled $y$-space and parabolic m.f.s, when the precedent output value $y_{\mathrm{n}}$ satisfies $a_{k-1} \leq y_{n} \leq a_{k}$, the $(n+1)^{\text {th }}$ iteration is

$$
x_{n+1}=\frac{\left(a_{k-1}\left(y_{n}-a_{k-1}\right)^{2}+\left(a_{k-1}+c\right)\left(y_{n}-a_{k-1}\right)^{2}\right)}{\left(y_{n}-a_{k-1}\right)^{2}+\left(y_{n}-a_{k-1}\right)^{2}},
$$

The computation results illustrate the difference in behaviour between the fuzzy tent map iteration and the crisp iteration with CoFT applied only to the values produced by the crisp tent map iteration. Figure 2 shows the iteration of the tent map with the parameters $s_{1}=1.91$; $x_{1}=2.6178 ; s_{2}=-1.91$ and the initial condition $x_{0}=1.5215$. After applying a CoFT to the output of the crisp tent map using the same membership functions as in Figure 1, $a_{0}=0 ; \quad a_{1}=1 ; \quad a_{2}=2 ; \quad a_{3}=3 ; \quad a_{4}=4 ; \quad a_{5}=5$, the transformed output, denoted $y^{*}[n]$ was compared to the sequence generated by the crisp tent map, $y[n]$. The time series of the difference $y^{*}[n]-y[n]$ is shown in Figure 3; while chaotic, this difference time series is strongly correlated with the initial one, $y[n]$, with a correlation coefficient close to 1 . However, the CoFT has a benefic effect, making more uniform the distribution of the values in the time series (i.e., a more uniform coverage of the output values.) The effect of applying the CoFT is also evidenced in the third graph (phase plot) in Figure 4.

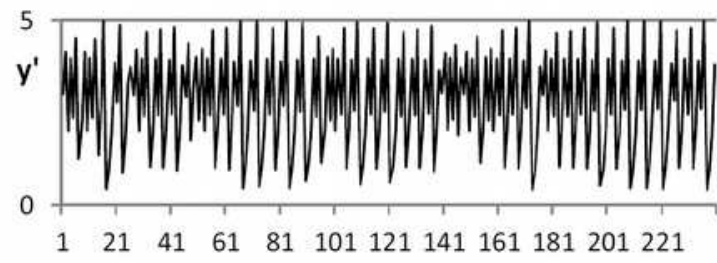

Figure 2. Time series generated with the standard tent map.

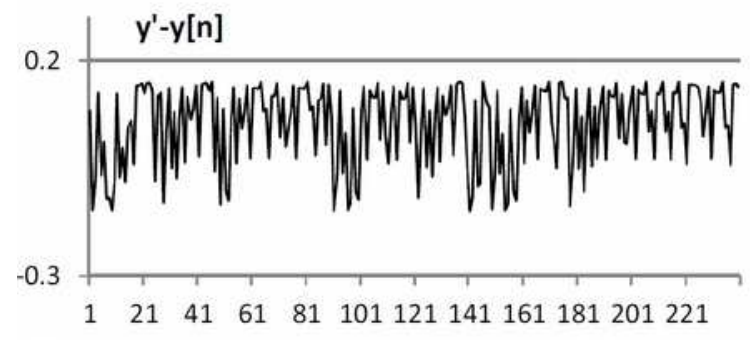

Figure 3. The difference time series. The parameters of the tent maps are given in the text. 


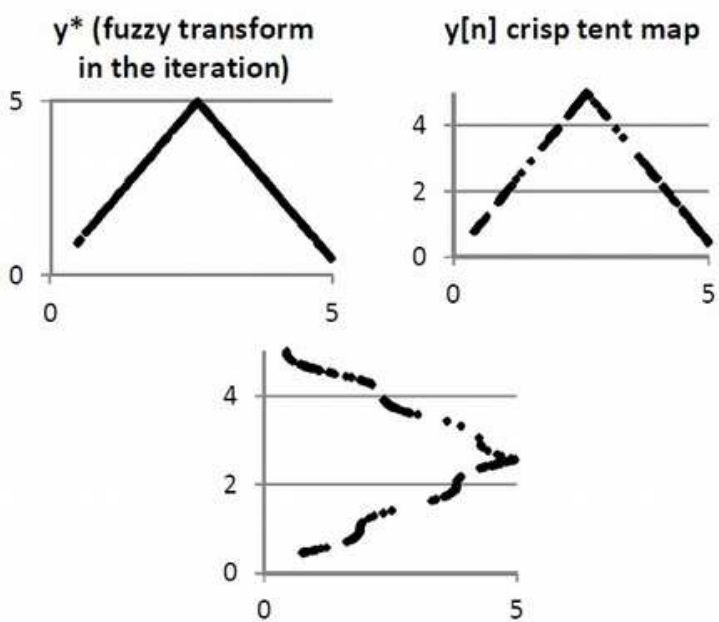

Figure 4. Effects of applying the CoFT to the series generated by the standard tent map

The results discussed subsequently are based on iteration as in (10) for the case in (11), in addition, for iteration as in (9). Figure 5 represents the time series generated by the fuzzy tent map described by (8) and (10), with the same parameters and initial value as above. Not only visually the times series in Figures 2 and 5 are different, but their covariance has the value Covar $=0.0495$ and their correlation coefficient is Corr $=0.0285$, demonstrating the strong effect the CoFT has in the fuzzification of the tent map. (This also explains why the difference time series in Figure 3 is correlated with the original time series).

$y^{*}$ (fuzzy transform in the iteration)

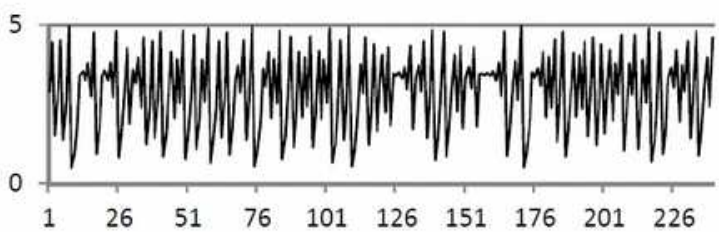

Figure 5. Series generated with the tent map obtained by the fuzzy transform of the $x$ coordinate.

Finally, the Fourier spectrum of the time series generated by the fuzzy tent map is shown in Figure 6; the spectrum (arbitrary units for the amplitude, vs. frequency bins) has some similarity with the $1 / f$ noise for higher frequencies, but not for the lower ones.

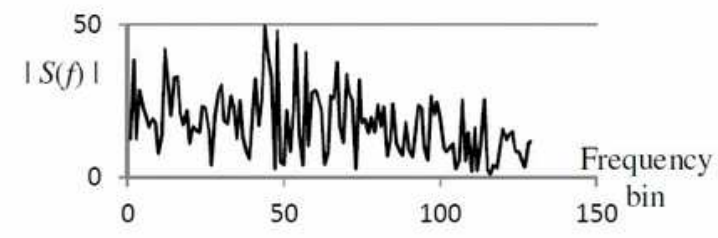

Figure 6. Fourier spectrum of a sequence of samples generated by the fuzzy tent map iteration
The above results support the notion that there is a benefit in using CoFT in the loop (iteration) of a tent map, in terms of enriching the range of chaotic regimes of the tent map. However, a thorough investigation, using a variety of tools [20] should be carried out before deciding on the suitability of specific fuzzy tent map implementations in applications.

\section{CoFT Application to Chaotic Series Prediction}

Various applications of the CoFT can be imagined, ranging from the optimization of nonlinear classification to decision making. One of them, the trimming of the chaotic processes, has been shown in the previous Section. Here we show an elementary example of a new method of improving the time series prediction, based on the CoFT in conjunction with a standard LPC predictor.

There are so numerous methods based on fuzzy systems, neural networks, and neuro-fuzzy systems for prediction of time series in the literature that even their basic comparison becomes a terrible feat. As a matter of example, only the researches using fuzzy methods in predicting Mackey-Glass time series produces (Google Scholar) more than 3100 papers. Methods used range from recurrent neural networks [6], to evolving radial basis function networks [4], to adaptive neuro-fuzzy inference system (ANFIS) with self-feedbacks [22], and to ensembles of ANFIS models [9], to name just a few recent works.

Our aim is not to show that a method based on a combination of CoFT and a fuzzy or neurofuzzy predictor can improve the results obtained by the sophisticated current methods of prediction, but only to show that the CoFT may improve a standard predictor already determined for some specified time series. For this purpose, we consider one of the best known nonlinear time series, the logistic series, determine for it the best linear predictor (LPC) as generated by the corresponding MATLAB toolbox, and then try to improve it using an adapted CoFT. The logistic series was generated with the recursion

$$
x[n]=3.64 \times x[n-1] \times(1-x[n-1])
$$

with the seed (initial value) $x[1]=0.7$. An LPC model of order 12 was identified for the first 150 samples of the logistic process (12) and the total square error of the model was computed. 
Then, an elementary CoFT transform was used to modify the input to the LPC model determined. The CoFT was represented by a basic TSK system with only three triangular input m.f.s, as shown in Figure 7(a). The three output singletons of the TSK system are respectively $0, V$, and 1 .

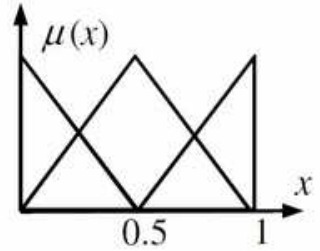

(a)

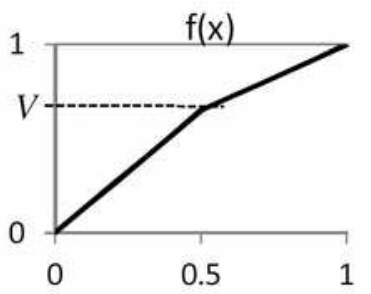

(b)
Figure 7. The basic CoFT with parameter $V$.

The value of the parameter $V$ was the only adjustable parameter of the CoFT, which is obviously represented by a piecewise transform $x \rightarrow x^{\prime}$ with the graph shown in Figure 7(b). The transformed values $x^{\prime}[n]$ were fed to the LPC determined on the original series. The value of $V$ was adjusted in a loop, in an elementary search. For every value of $V$ between 0 and 1 , the values of the square error between the output of the LPC and the original series was computed and compared with the error of the LPC on the original series. When the new error was less than the original, $V$ was stored and then the optimal choice for $V$, providing the minimal error, was the result of the optimization. The optimization produced the value $V=0.525$, with the error 2.1344, compared with the initial error of the LPC, 2.1607. The original series and the original LPC model are shown in the upper panel of Figure 8, while the result obtained with the CoFT is shown in the lower panel of the same figure. This extremely simple example demonstrates that the CoFT method may help in various modeling and prediction problems, as discussed in the next Section.

The method was also tested on time series of noise detected on Social Networks and on time series of tremor signals, the last ones being known to have a chaotic component [5]. The preliminary results shown sometimes similar improvements to those illustrated above, but more work is needed for improving the method.

\section{Discussion and Conclusions}

A general definition of fuzzy transformation of the Euclidean space was suggested and the properties of the transform were investigated; then the definition was exemplified. The transforms have an interpolative nonlinear effect, which is periodical under uniformly distributed m.f.s in the transformed space. When the transform is incorporated in the application performed by tent maps, it produces a 'fuzzy tent map' with more intricate behaviour and with a larger number of parameters available for adjustment in
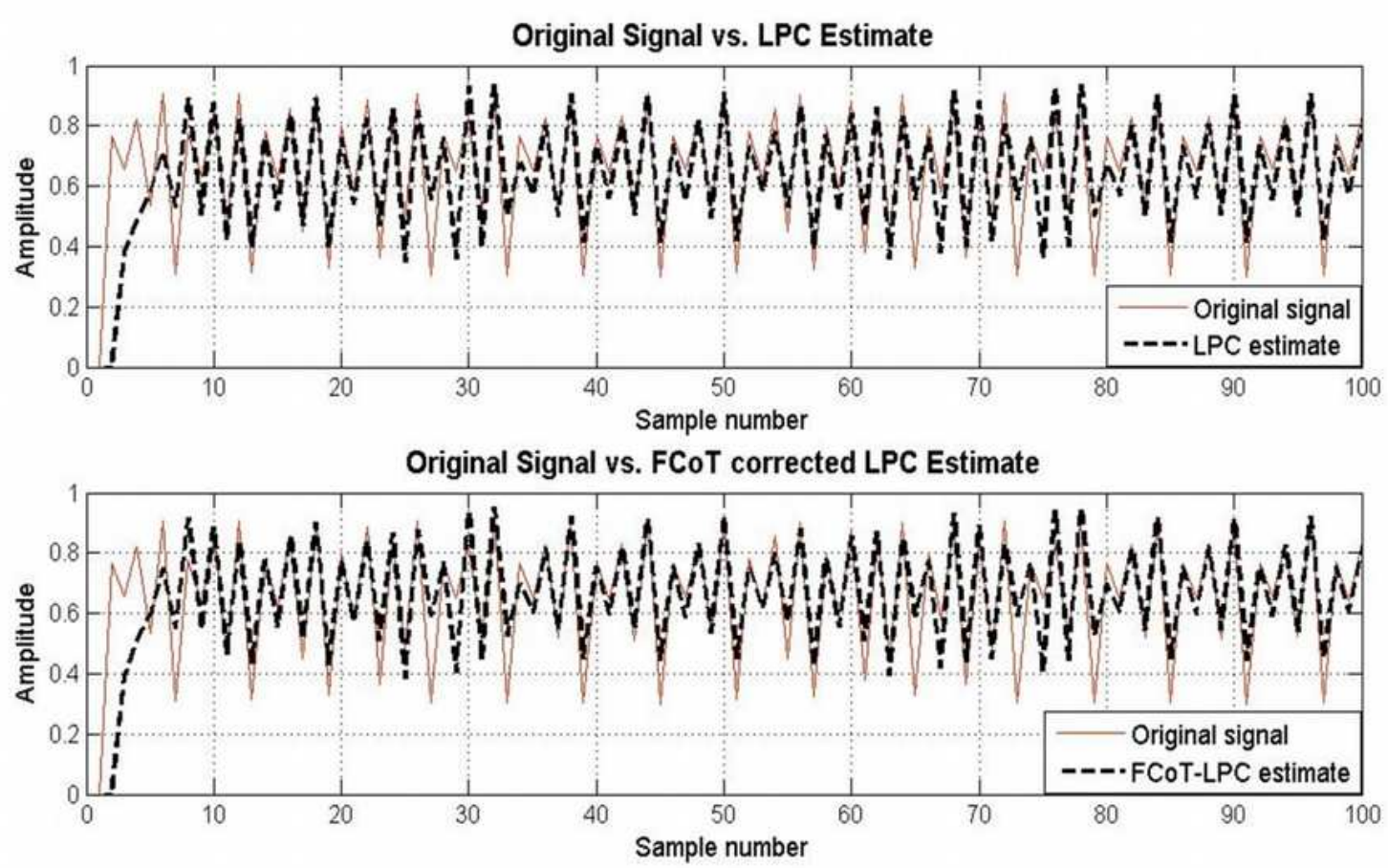

Figure 8. Applying the CoFT transform in combination with an LPC predictor (first 100 samples). 
prediction and modelling. When the transform is external to the iteration, its effect is null in terms of enriching the dynamics, yet it may be useful for equalizing the distribution probability (histogram) of the generated series. It was determined that, for the same initial condition and for the same basic tent map, the fuzzy tent map produces time series that are non-correlated with the ones generated by the corresponding crisp tent map.

While simple iterated functions are used frequently to model natural processes, where modelling means mimicking some of the properties of the natural time series, such models may have limited power. For example, with a single parameter, the class of standard tent maps is poorly equipped for adaptation in modelling and predicting chaotic time series. An alternative is to employ polynomial iterated functions, or piecewise polynomial tent maps, but the computations become more intricate and the intuition of the effect of the polynomial coefficients is poor. In this paper, a relatively simple alternative was suggested, using coordinate transformations before applying the tent map. The advantage of this type of tent map is that the space deformation is an intuitive concept and meaning may be attached to such deformations. For example, in social and economic processes, deforming the opinion of the population groups may induce modified social dynamics.

The coordinate fuzzy transforms may find a variety of applications in model and control trimming for critical applications such as those of implants [5], in adaptive assessment for decision making as for the problem in [25], and in improving classification. Fuzzy tent maps obtained by the fuzzy transform may be useful in secure communications, in protecting the hardware, and in generating a large number of signals for communication equipment testing.

Also, an appealing application could be the mapping of a social process, such as the response of social networks to events, into another of the same type, assuming that the two processes involve populations with similar behaviours that can be mapped by a fuzzy transform, where the fuzzy transform reflects the relation between the different subjectivity of the individuals in the two populations. Also, if fuzzy mappings prove in further work to transform one noise time series on Social Networks in another noise, this may help understand the characteristics of these noise processes.

\section{Acknowledgements}

The work for the applicative section of this research related to time series and their predictors, as well as the publication of the paper were supported by NATO-SPS grant 984877/G4877. The author acknowledges the support. The funders had no role in the choice of the topic or in the actions leading to the results, and no influence on the preparation of the manuscript. The author keeps available the code and worksheets used in this research to anyone asking for them at the address hteodor@etti.tuiasi.ro. Data are self-generated.

\section{REFERENCES}

1. BODLAENDER, H. L., J. Van LEEUWEN, Simulation of Large Networks on Smaller Networks. Information and Control, vol. 71, 1986, pp. 143-180.

2. CRAMPIN, M., B. HEAL, On the Chaotic Behaviour of the Tent Map. Teaching Mathematics Applications vol. 13(2), 1994, pp. 83-89.

3. DAŇKOVÁ, M., M. ŠTĚPNIČKA, Fuzzy Transform as an Additive Normal Form. Fuzzy Sets and Systems, vol. 157(8), Apr. 2006, pp. 1024-1035.

4. DU, H., N. ZHANG, Time Series Prediction using Evolving Radial Basis Function Networks with New Encoding Scheme, Neurocomputing, vol. 71(7-9), March 2008, pp. 1388-1400.

5. GEMAN, O., H. N. TEODORESCU, C. ZAMFIR, Nonlinear Analysis and Selection of Relevant Parameters in Assessing the Treatment Results of Reducing Tremor, using DBS Procedure. Proceedings IEEE International Joint Conference on Neural Networks (IJCNN), Budapest, Hungary, Jul 25-29, 2004, vol. 14, pp. 2461-2466.

6. GRAVES, D., W. PEDRYCZ, Fuzzy Prediction Architecture using Recurrent Neural Networks. Neurocomputing, vol. 72(7-9), Mar 2009, pp. 1668-1678.

7. GUEGAN, D., Chaos in Economics and Finance. Annual Reviews in Control, IFAC, vol. 33(1), 2009, pp. 89-93.

8. HSIEH, D. A., Chaos and Nonlinear Dynamics: Application to Financial 
Markets. The Journal of Finance, Vol. 46, no. 5, Dec 1991, pp. 1839-1877.

9. MELIN, P., J. SOTO, O. CASTILlO, J. SORIA, A New Approach for Time Series Prediction using Ensembles of ANFIS Models. Expert Systems with Applications, vol. 39, 2012, pp. 3494-3506.

10. PERFILIEVA, I., Fuzzy Transforms. In J.F. Peters et al. (Eds.): Transactions on Rough Sets II, LNCS 3135, Springer, Berlin Heidelberg 2004, pp. 63-81.

11. PERFILIEVA, I., Fuzzy Transforms: Theory and Applications. Fuzzy Sets and Systems, Vol. 157(8), 2006, pp. 993-1023.

12. PERFILIEVA, I., V. NOVAK, V. PAVLISKA, A. DVORAK, M. STEPNICKA, Forecasting Time Series using Fuzzy Transform. http://www.neural-forecasting -competition. com/downloads/NN3/methods/11_NN3_Pe rfilieva_NN3.pdf Apr 3, 2015.

13. PERFILIEVA, I., Fuzzy Transforms in Image Compression and fusion. Acta Mathematica Universitatis Ostraviensis, vol. 15(1), 2007, pp. 27-37.

14. PERFILIEVA, I., Fuzzy Transforms and Their Applications to Image Compression. Fuzzy Logic and Applications. Lecture Notes in Computer Science Vol. 3849, 2006, pp. 19-31.

15. ROGERS, T. D., D. C. WHITLEY, Chaos in the Cubic Mapping. Mathematical Modelling, vol. 4, no. 1, 1983, pp. 9-25.

16. PALACIOS, A., Cycling Chaos in OneDimensional Coupled Iterated Maps. Int. J. Bifurcation and Chaos, Vol. 12, 2002, no. 8, pp. 1859-1868.

17. DE OLIVEIRA J. A., E. R. PAPESSO, E. D. LEONEL, Relaxation to Fixed Points in the Logistic and Cubic Maps: Analytical and Numerical Investigation. Entropy, vol. 15, 2013, pp. 4310-4318.
18. TEODORESCU, H.-N., A. KANDEL, M. SCHNEIDER, Fuzzy Modeling and Dynamics, Fuzzy Sets and Systems Vol. 106, Aug 16 1999, no. 1, pp. 1-2.

19. TEODORESCU, H.-N., Pattern Formation and Stability Issues in Coupled Fuzzy Map Lattices. Studies in Informatics and Control, ICI Publishing House, vol. 20, 2011, no. 4, pp. 345-354.

20. TEODORESCU, H.-N., Characterization of Nonlinear Dynamic Systems for Engineering Purposes - A Partial Review. International Journal of General Systems, vol. 41, 2012, no. 8, pp. 805-825.

21. TEODORESCU, H.-N., On Fuzzy Sequences, Fixed Points and Periodicity in Iterated Fuzzy Maps. International Journal on Computers Communications \& Control, vol. 6, no. 4, 2011, pp. 752-763.

22. VAIRAPPAN, C., H. TAMURA, S. GAO, Z. TANG, Batch type Local Search-based Adaptive Neuro-fuzzy Inference System (ANFIS) with Self-feedbacks for Timeseries Prediction. Neurocomputing, vol. 72, nos. 7-9, Mar 2009, pp. 1870-1877.

23. VLAD, A., A. LUCA, O. HODEA, R. TATARU, Generating Chaotic Secure Sequences Using Tent Map and a Running-Key Approach. Proceedings Romanian Academy, Series A, Vol. 14, Special Issue 2013, pp. 295-302.

24. YI, X., Hash Function based on Chaotic Tent Maps, Circuits and Systems II: Express Briefs, IEEE Trans., vol. 52(6), 2005, pp. 354-357.

25. ZAVADSKAS, E. K., Z. TURSKIS, J. ANTUCHEVICIENE, Selecting a Contractor by Using a Novel Method for Multiple Attribute Analysis: Weighted Aggregated Sum Product Assessment with Grey Values (WASPAS-G), Studies in Informatics and Control, ISSN 12201766, vol. 24, no. 2, 2015, pp. 141-150. 